\title{
CT protocols: balancing radiation dose and image quality
}

\section{Richard Fotter}

(C) Springer-Verlag 2011

\section{Background}

In recent years, the use of CT has increased rapidly both in adults and children. This development is of special concern for the paediatric radiologist, since ionising radiation carries a higher risk in children compared with adults. This is due to higher tissue radio-sensitivity and longer life expectancy. Therefore, the development of age-adjusted or weightadjusted CT protocols in children is of major importance. Dose reduction, by obtaining the lowest acceptable (appropriate) image quality for answering the clinical questions, has to be the central goal of such efforts.

Several articles have been published in recent years giving recommendations on dose-reduction techniques in paediatric CT, but only sparse information is available regarding the resulting image quality. Furthermore, there is no definition of what constitutes appropriate image quality in paediatric CT, in particular with regard to age, body region and clinical setting. CT protocols for children were primarily based on empirical data from adult protocols; in many cases, only tube current (mAs) was reduced, while either keeping the voltage $(\mathrm{kVp})$ at adult level or reducing it without knowledge of the resulting image quality, in particular low-contrast detectability.

It is well known that CT image quality can be evaluated by mathematically measurable quality indices such as

Disclaimer The author's department has a research contract with Toshiba Medical Systems, Austria.

R. Fotter $(\bowtie)$

Department of Paediatric Radiology, Medical University Graz,

University Hospital,

Auenbruggerplatz 34,

Graz, Styria 8036, Austria

e-mail: richard.fotter@medunigraz.at quantum noise, signal-to-noise ratio, contrast-to-noise ratio, dose efficiency and so on. Even at the beginning of CT application in children in the late 1970s, it was well known that the greatest challenge in paediatric $\mathrm{CT}$ is to cope with low contrast and lack of fat, in particular in infants and young children. But, even today, very limited data are available on what is an appropriate low-contrast detectability.

Our vision was to optimise paediatric CT technique, in particular radiation dose and image quality, based on a systematic scientific approach using semi-anthropomorphic phantoms for different age groups. The idea was to challenge the "top-down" approach (empirical extrapolation of adult protocols) by developing a reproducible and widely applicable standard via a "bottom-up" approach. For this purpose, phantoms were developed and a project was started.

\section{Objective}

Our goal was to develop evidence-based CT protocols for children and young adults, balancing radiation dose and image quality, irrespective of scan type and CT technology. This should be based on mathematically measurable quality indices and low-contrast detectability by age and body-part, and based on phantom studies.

\section{Materials and methods}

CT equipment All measurements were done with the AquilionOne 320-row multi-detector CT (Toshiba Medical Systems, Tochigi, Japan). This scanner has a 320-row, 0.5-mm detector and permits scanning at a speed of $0.35 \mathrm{~s} /$ rotation. Adoption of the 160-mm wide area detector makes it possible 
to select slice thickness for scanning at $0.5,1,2,3,4,5$ or $8 \mathrm{~mm}$, depending on the purpose of the scan. The volume scan mode allows a volume of $16 \mathrm{~cm}$ in the $z$-axis to be scanned in one rotation. In addition, helical scan modes using 64, 32 or 16 rows are available.

Phantoms Three semi-anthropomorphic chest and three semi-anthropomorphic abdominal phantoms of different antero-posterior and transverse diameters, all $200 \mathrm{~mm}$ long, for three age groups, were developed and used: $100 \times 150 \mathrm{~mm}$ transverse diameters to represent the body of a 10-month-old infant, $150 \times 225 \mathrm{~mm}$ to represent a 7.5-year-old, and a $200 \times$ $300 \mathrm{~mm}$ to represent a 16 -year-old. The phantoms consist of tissue-equivalent material and have a central hole for inserts of $50 \mathrm{~mm}$ diameter (smallest phantom) and $80 \mathrm{~mm}$ diameter (intermediate and large phantoms). The inserts contain spheres of 2, 3, 5 and $6 \mathrm{~mm}$; the $80-\mathrm{mm}$ insert contains additional $8-\mathrm{mm}$ spheres. The density differences of the spheres to the background are $-5,-10,-20$ and -50 Hounsfield units. The CT numbers of the simulated organs correspond to those of the human body at $120 \mathrm{kVp}$. Phantoms were in the iso-centre of the scanner.

Scan protocols $\mathrm{CT}$ images of the chest and abdominal phantoms with variable $\mathrm{kVp}$, mAs and scan mode (volume, and 32- and 64-helical) were acquired. For each scan mode and $\mathrm{kVp}(80,100,120), \mathrm{mAs}$ was varied. Rotation time was $0.5 \mathrm{~s}$, in case the desired $\mathrm{mAs}$ could not be achieved by further increasing the $\mathrm{mA}$, the rotation-time was increased instead. A standard reconstruction filter-function was used for all scans. Standard reconstructed slice thickness of $5 \mathrm{~mm}$ for the abdomen and $3 \mathrm{~mm}$ for the chest was used with increments of $1 \mathrm{~mm}$.

Dose measurements All phantoms had central holes, where two $10-\mathrm{cm}$ pencil ionisation chambers were inserted. The measured dose-values were corrected for temperature and air pressure. Radiation dose was calculated as $\mathrm{mGy} \cdot \mathrm{cm}$.

Quality measurements These were done objectively (mathematically) and subjectively (determination of low-contrast detectability). Objective measurements (calculated image quality indices) were the radiation dose versus pixel noise relationship, and the radiation dose versus contrast-to-noise ratio relationship. Subjective evaluation was done by three readers with different paediatric $\mathrm{CT}$ experience, who assessed low-contrast detectability on a three-grade scale.

\section{Results}

The results of subjective and objective evaluation of the phantom studies simulating paediatric chest and abdominal CT in three different age groups showed that objective measurements alone form only a basis. Only when matching these with the results of subjective evaluations (nominal standards of low-contrast detectability), is it possible to define the minimum acceptable CT image quality for a specific clinical setting, and hence the maximum achievable radiation dose reduction.

\section{Discussion}

Derivable results from these phantom studies may form the basis for developing age-adjusted paediatric CT protocols incorporating $\mathrm{kVp}, \mathrm{mAs}$, scan mode and reconstruction filter function. One limitation to our approach might be that we used only a limited number of phantom sizes (simulating three age groups). Nevertheless, from a practical point of view, the results allow protocol development for key age groups, which can then be extrapolated for smaller and larger patients. A second limitation is that the phantom studies were done exclusively with low contrast inserts, so the derived protocols are not appropriate for $\mathrm{CT}$ angiography.

\section{Conclusion}

Evidence-based paediatric CT protocol development balancing image quality and radiation dose is possible, and the developed approach is widely applicable. Objective and subjective measurements must be matched. Noise and contrast-to-noise ratio alone are insufficient and not generally applicable; the optimum balance among noise, contrast and radiation dose form only a basis. Appropriate nominal standards of lowcontrast detectability have to be defined and matched with objective measurements. 